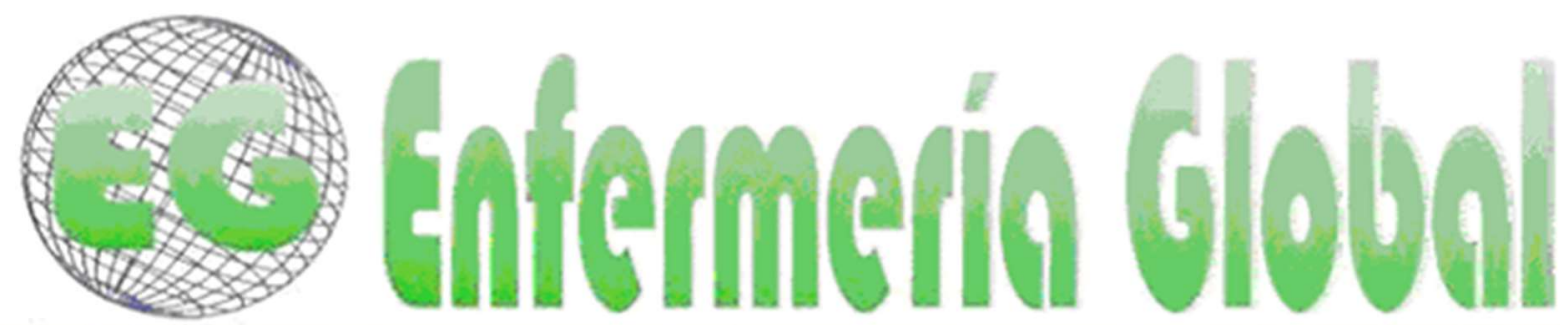

\title{
ORIGINALES
}

\section{Experiencia vivida del primer contacto con muerte en prácticas clínicas de estudiantes de enfermería}

Lived experience of first contact with death in nursing students' clinical practicum

\author{
Manuel de Jesús Hernández Ramírez ${ }^{1}$ \\ Erika Yovana González-Martí \\ Ashlye Melannie Fuentes Rodríguez ${ }^{1}$ \\ Suleika Alelí Carranza López ${ }^{1}$ \\ Violeta Compeán Padilla ${ }^{1}$ \\ Raúl Fernando Guerrero Castañeda ${ }^{2}$
}

1 Departamento de Enfermería, Centro de Ciencias de la Salud, Universidad Autónoma de Aguascalientes, Aguascalientes, México.

2 Departmento de Enfermería Clínica, División de Ciencias de la Salud e Ingeniería, Campus Celaya Salvatierra, Universidad de Guanajuato, Celaya, Guanajuato, México. drfernandocastaneda@hotmail.com

\section{https://doi.org/10.6018/eglobal.483631}

Recibido: 16/06/2021

Aceptado: 21/09/2021

\section{RESUMEN:}

Introducción: La muerte es un fenómeno complejo, las enfermeras en formación pueden enfrentarse al proceso de morir y muerte de personas, lo que puede generar diversas situaciones que representan sus experiencias vividas.

Objetivo: Comprender la experiencia vivida ante el primer contacto con la muerte durante prácticas clínicas de estudiantes de enfermería de una universidad pública.

Metodología: Estudio cualitativo con enfoque fenomenológico. Se llevó a cabo de octubre del 2020 a febrero del 2021. Selección de participantes por muestreo intencional con saturación de información con siete participantes. Recolección de datos a través de entrevista fenomenológica; los participantes emitieron su consentimiento informado. El análisis se llevó a cabo por las tres fases del método fenomenológico hermenéutico de Max Van Manen (descripción, interpretación, descripción más interpretación).

Resultados: Los estudiantes refieren haber estado frente al fenómeno en un estado de impotencia, bloqueo, incertidumbre y miedo; así como afrontaron la muerte de manera profesional y reflexiva para brindar cuidado de calidad.

Discusión: La muerte es un proceso natural, sin embargo, las primeras experiencias son diversas y pueden producir en los estudiantes un conjunto de emociones que condicionan su capacidad de actuar, el modo de adaptarse y observar las necesidades surgidas durante esta experiencia.

Conclusión: Las experiencias de los estudiantes de enfermería demuestran que tienen dificultades para afrontar la muerte, pero no fue un impedimento para brindar los cuidados necesarios; se considera que una preparación más profunda sobre el proceso de morir puede ser favorable para su actuar como profesional. 
Palabras clave: Muerte, Estudiantes de enfermería, Experiencia, Prácticas clínicas.

\begin{abstract}
:
Introduction: Death is a complex phenomenon, nurses in training may face the process of dying and death of people, which can generate diverse situations that represent their lived experiences.

Objective: To understand the lived experience of the first contact with death during clinical practicum of nursing students at a public university.

Methodology: Qualitative study with phenomenological approach. It was conducted from October 2020 to February 2021. Selection of participants by intentional sampling with information saturation with seven participants. Data collection through phenomenological interview; participants gave informed consent. The analysis was carried out through the three phases of Max Van Manen's hermeneutic phenomenological method (description, interpretation, description plus interpretation).

Results: The students refer to having been faced with the phenomenon in a state of helplessness, blockage, uncertainty, and fear; as well as facing death in a professional and reflective manner in order to provide quality care.

Discussion: Death is a natural process; however, the first experiences are diverse and can produce in the students a set of emotions that condition their capacity to act, the way of adapting and observing the needs that arise during this experience.

Conclusion: The experiences of nursing students show that they have difficulties in facing death, but it was not an impediment to provide the necessary care; it is considered that a deeper preparation on the process of dying can be favorable for their performance as professionals.
\end{abstract}

Key words: Death, Nursing students, Experience, Clinical practicum.

\title{
INTRODUCCIÓN
}

Enfermería vive día a día en su práctica la muerte de personas por algún padecimiento, dentro de sus funciones tiene la de apoyar y ayudar en el proceso de morir y el cómo se afronta la transición del fallecimiento de personas que cuida o pacientes y los seres queridos de este, es por ello que se espera una actitud cálida, favorable y de apoyo con el necesitado (1). La importancia de la formación académica de los futuros profesionales de enfermería destaca precisamente en fomentar el cuidado; a lo largo de su formación los estudiantes comienzan a observar diferentes fenómenos y se abren a reflexionar sobre ellos, lo cual hace que cuando viven la primera experiencia frente al término de la vida de una persona, surjan dudas y cierto temor al no saber cómo actuar ante la ejecución de la práctica clínica, ocasionando el carecer del disfrute de la experiencia al brindar cuidado ${ }^{(2)}$.

La muerte es un fenómeno que resulta de la extinción del proceso homeostático en un ser vivo y con ello se dirige al fin de la vida; la muerte es el cese permanente de la conciencia del organismo en todas sus formas, así mismo de la pérdida de las funciones del organismo como un todo (3). Por otro lado, Weismann hace una distinción entre causas externas e internas de muerte, señalando que con la edad hay ciertos cambios en los tejidos que consumen su funcionamiento acabando por direccionar a lo que se denomina una muerte normal, o bien conducen indirectamente a la muerte, al hacerlo incapaz de resistir ante influencias patológicas perjudiciales ${ }^{(4)}$.

Este concepto de muerte es visto de manera diferente por el profesional de enfermería, ya que este se vincula directamente con sus ideas, quehaceres, costumbres, vivencias y experiencias; por ende, las actitudes ante la muerte son distintas en cada persona ${ }^{(5,6)}$. En la actualidad, existe rechazo y negación ante el tema de la muerte, haciendo que el profesional de la salud ponga una barrera y evite a toda costa lidiar con ella (7-9); estudios muestran que existen actitudes que se 
replican en los profesionales, algunos de ellos son: distanciamiento, evitación, huida y disociación emocional $(1,5,10)$.

Durante el desarrollo de la práctica clínica los estudiantes probablemente no cuenten con las herramientas necesarias para sentirse lo suficientemente capacitados para enfrentar la muerte de un paciente ${ }^{(10)}$, ya que es un evento nuevo y difícil de enfrentar, por esta razón se dio paso a la intención de comprender la primera experiencia vivida del estudiante sobre el fenómeno de la muerte, pues la experiencia vivida impacta en la formación del enfermero en su vida personal y relacional y académica ${ }^{(11)}$.

El objetivo de este estudio fue comprender la experiencia vivida del primer contacto con muerte en prácticas clínicas de estudiantes de enfermería.

\section{MÉTODO}

Se trata de un estudio cualitativo con enfoque fenomenológico. La fenomenología como filosofía y como método de investigación aborda la búsqueda de significados y esencias de las experiencias vividas ${ }^{(12,13)}$, permite develar la experiencia desde la conciencia, Max Van Manen retoma los conceptos de la fenomenología de Husserl y menciona que la fenomenología como método no se limita únicamente a un enfoque ni a una simple descripción o alternativa, sino a la búsqueda de la esencia de la conciencia en experiencias significativas ${ }^{(14)}$, por ello y con el objetivo de comprender las experiencias vividas del estudiante se retomó el referente metodológico de Max Van Manen ${ }^{(15)}$, lo que permitió una aproximación a los fenómenos que son esenciales en el cuidado de enfermería en el proceso de morir y la muerte.

El escenario del estudio fue una universidad pública del estado de Aguascalientes, México, en el periodo de octubre del 2020 a enero del 2021. Para la selección de los participantes los criterios fueron: estudiantes de la licenciatura en enfermería que cursaron entre tercer y octavo semestre que manifestaron haber tenido su primer contacto con la muerte de una persona durante la práctica clínica; para seleccionar a los participantes se envió una invitación virtual a través de un mensaje escrito a los líderes de clase en grupos conversacionales en donde se indicó el nombre de la investigación, objetivo, criterios de selección e investigadores, así los participantes con los criterios contactaron a los investigadores para manifestar su deseo de participar.

Se realizó un muestreo intencional obteniendo una muestra de siete estudiantes de enfermería de acuerdo con los criterios de selección establecidos, alcanzando la saturación de información, de tal manera que por medio del análisis se obtuvieron datos repetitivos y relevantes clasificados en diversas unidades de significado para comprender el fenómeno de la experiencia vivida y agruparlas en temas ${ }^{(16,17)}$.

La recolección de datos se realizó mediante una entrevista fenomenológica individual(18), a través de la pregunta orientadora: "¿Cómo fue su experiencia vivida ante el primer contacto con la muerte en prácticas clínicas?” y utilizando algunas preguntas de rescate. La entrevista fenomenológica permitió un diálogo con los participantes, esta a su vez facilita el encuentro con el fenómeno vivido determinando las características de la experiencia vivida ${ }^{(18)}$. 
Las entrevistas fueron realizadas por dos estudiantes investigadores que fueron entrenados para ello, además contaron con la supervisión de dos investigadores expertos en métodos cualitativos después de cada entrevista, las cuales se basaron en la empatía por parte de los entrevistadores y a su vez, no surgió la condición de subordinación debido a que no eran compañeros directos de los investigadores y que no se impartía clases a ningún participante. Las entrevistas fueron audio grabas previo consentimiento de los participantes. El tiempo promedio de duración de la entrevista fue de 28 minutos. Debido a la contingencia COVID-19 se realizaron cinco entrevistas en modalidad virtual a través de la plataforma Google Meet ${ }^{\circ}$ y dos de manera presencial, manteniendo protocolo de seguridad emitido por la Secretaría de Salud de México.

Todas las entrevistas fueron transcritas de forma fiel por los investigadores en Microsoft Word®; una vez transcritas se realizaba un primer análisis por cada investigador, el análisis fue de tipo artesanal a través de las fases del método fenomenológico-hermenéutico según Max Van Manen que consta de tres fases: descriptiva (recoger la experiencia), interpretativa (reflexión acerca de la experiencia) y descripción más Interpretación (escribir-reflexionar acerca de la experiencia y la creación del texto fenomenológico) ${ }^{(19)}$. Las entrevistas se trabajaron en tablas en Microsoft Word® destacando unidades de significado, temas y síntesis de la esencia de las unidades. Los temas fueron considerados por consenso de los investigadores.

El protocolo de investigación fue aprobado por la Academia de Investigación del Departamento de Enfermería del Centro de Ciencias de la Salud de la Universidad Autónoma de Aguascalientes, obteniendo el código AEI-09-20 que contó con los lineamientos básicos para su desarrollo, además se cumplió con los criterios éticos del Reglamento de la Ley General de Salud en Materia de Investigación para la Salud. Los participantes emitieron su consentimiento informado, cuidando en todo momento la confidencialidad de los participantes brindándoles un código como identificación (20).

\section{RESULTADOS}

Tomando en cuenta las características de los estudiantes, el $57 \%$ son de sexo femenino y el $43 \%$ restante de sexo masculino, el rango de edad se encuentra entre los 20 y 31 años, con un promedio de edad de 23 años, además todos los participantes cursaban entre tercero y octavo semestre de la licenciatura en enfermería. Emergieron cinco temas con sus respectivas unidades de significado, como resultado del análisis, los cuales son presentados a continuación:

1. Emociones encontradas ante el impacto de la muerte. Estas se describen a partir de la confirmación del fallecimiento de la persona, al verse inmersos en el fenómeno de muerte los estudiantes manifiestan preocupación sobre cómo se dará esta noticia, refieren que es impactante, a tal grado de haber llorado o querer hacerlo. Los participantes describen que es como encontrarse en un "estado de shock"; expresado como una situación "neutra con diversas emociones al mismo tiempo", "vacío", "tristeza", "impotencia", "miedo", este último no a la muerte sino al proceso que se deriva de ella; además de "desesperación", "frustración", una confusión de sus sentimientos y un impacto sobre lo percibido; a consecuencia de esto evitaban actuar de manera espontánea, pensaban que por expresar sus emociones podrían ser juzgados, haciendo percibir una barrera que les impide actuar con confianza, a sí 
mismo inmaduros o poco preparados para afrontar la muerte. Como se menciona en los siguientes discursos:

"Me fui a un rincón de la sala a llorar para mí, para mí, porque incluso los médicos se me quedan viendo raro, así como de esta qué [...] me fui al rincón, porque tenía vergüenza no quería que me viera nadie llorando porque pues, para mi..., no sé, o sea, me iban a juzgar." E2F.

"Eh respecto a esa muerte creo que fue la que más me impactó, creo en ese momento pues no me sentía, lo suficientemente preparado y maduro a la vez de hacer acciones así." E4J.

"No sé cómo describir ese sentimiento entre alivio y tristeza, fue un choque de emociones." E50.

"Yo creo que todo se resume en miedo. El miedo es el que no, [...] no te deja, siento que es así como una barrera enorme que no te deja hacerlo, como si tu quisiera hacerlo, pero eso te hiciera para atrás [...] fuera una molestia para la señora" E1L.

2. Incertidumbre ante la muerte. Se puede identificar que los estudiantes se encontraban en un estado de no comprender lo que ocurría al ser el primer contacto con el fenómeno, surge la incertidumbre acompañada del impacto del suceso, comenzaron a surgir interrogantes al presenciar el cese de la vida, sobre qué cuidados tenían que proporcionar al paciente o el actuar "correcto" que la situación requería, así mismo, se realizaban preguntas del “¿Por qué sucede?”, “¿Qué sucede con la familia del paciente?”, “¿Qué pasa con el personal que lo está presenciando?”, y "¿Por qué actúan de la manera en que lo hacen?"; además, indican haberse percatado de la reacción del personal de salud y algunos familiares que presenciaron el suceso ocasionando que influyera en su actuar. Como lo expresan los siguientes discursos:

"Pues fue muy extraño, o sea, para ser la primera vez creo que sí me afectó mucho, porque fue algo que yo no esperaba y yo no estaba enterada de nada" E2F.

"Empezaron los gritos y todo por parte de los familiares, la enfermera también se quedó en shock [...] se metió al baño a llorar, [...] entonces pues si te das cuenta de del impacto que tiene una muerte" E4J.

"Con cierto punto de incertidumbre [...] ¿Y ahora qué hago? ¿Cómo yo debería actuar? [...] me costó trabajo asimilar esa parte de decir se murió y ahora ¿Qué voy a hacer? o ¿Qué sigue?” E50.

"Creo que jeje fueron de las cosas más difíciles de mi vida en ese momento [...] meter algodón en todo orificio del cuerpo humano [...] es una experiencia rara, decir, me acuerdo que tenía esa duda de decir y ¿No le dolerá? Fue un pensamiento que tuve de decir y ¿No le dolerá a la señora?” E50

3. La relación enfermera-docente-estudiante. En este tema surgieron dos situaciones opuestas, por un lado, el estudiante percibe cómo fue la respuesta del personal de 
salud ante el suceso, expresando que ellos difieren en la forma en cómo actuaron los profesionales, pues consideran que los cuidados hacia la persona fallecida son distantes y esto lo ven los profesionales como algo normal pero frío, así mismo, refieren la actitud de los docentes, mencionando que estos comentaron la frase "va a ser tu día a día", dando como resultado la "normalidad". Los discursos siguientes expresan esta postura:

"No, el personal, realmente no me explicó nada, simplemente me dijeron se va a morir, no queremos que se muera en el turno y ya." E5O.

"Si me llegó el sentimiento al hablarlo con mi docente y pues ella me dijo que era, era normal, o sea era algo que iba a ser parte de mi día a día, sin embargo, no me dio tiempo me dijo que regresara al servicio." E6E.

Además, emergen por otro lado, situaciones de parte de las enfermeras del área hacia el estudiante en donde por sus acciones en el momento de la muerte, los estudiantes lograron encontrar tranquilidad, orientación o apoyo y que en ese momento figuraron como guía, ya que cuentan con más conocimientos y experiencia para afrontar de manera adecuada dicha situación, además señalaron a sus compañeros como una fuente de confianza que los ayudó a asimilar y controlar sus emociones antes y durante el fenómeno. Como lo demuestra en los siguientes discursos:

"Pero lo que más me causó impacto fue ver a la enfermera acercándose con la madre diciéndole ¿cómo quieres que, que se llame tu hijo?, y así fue como, como, como un rayito de luz." E2F.

"El profe [...] tiene un poco más de experiencia de conocimiento ante estas escenas, [...] fue el que nos iba diciendo: haz esto, eh nos tranquilizaba" E4J.

4. Sentido de profesionalismo ante el fenómeno. Los estudiantes se describen desde una perspectiva profesional de enfermería; guiándose a través de sus valores como: respeto, empatía, confidencialidad y el actuar con humanismo, indicaron sentir satisfacción y emoción por las intervenciones realizadas en el cese de la vida, destacaron las actitudes propias y de los enfermeros hacia el paciente fallecido y a los familiares que están viviendo un duelo o el "dolor de la pérdida" que fueron de gran contribución moral haciéndoles sentir de cierta manera consuelo y alivio al ver algo positivo dentro de todo lo vivido y/o encontrar resignación por la pérdida. Como lo expresan los siguientes discursos:

"El hablar con ella y estar cerca... este... de ella [...] me hizo sentir bien o sea como que no estaba realmente solo si faltaba su familiar [...] sentía que de cierto modo estaba yo ahí con ella". E6E.

"Cuando porto el uniforme si me creo, trato de creerme mucho, eso de que soy enfermera y... yo tengo que ayudar a los pacientes o hacerlos fuertes a ellos." E1L.

"Me sentía con mucha satisfacción por la persona porque yo sabía que lo estaba tratando con mucho respeto sabía que lo estaba haciendo por un bien 
[...] más que nada que con todas esas emociones buenas que yo tenía, con toda esa motivación de hacerlo sentir bien." E7A.

5. Reflexión memorial del fenómeno de morir. El compartir sus experiencias les hizo reflexionar acerca de poder mejorar sus acciones y que enfermería les ha aportado valor para afrontar la situación. Además, refieren que el hecho de recordar los hizo transportarse al momento y revivir los sentimientos que los invadió en dicho suceso, narran que vivir esta experiencia dejó algo significativo; destacando que lo que más recordaron de ese momento fue el proceso de amortajamiento, los actos de la persona antes de su muerte y la forma en cómo reaccionó la familia. Indican que la situación cambia "de un segundo a otro", que ese momento fue "rápido" y no pudieron actuar o responder a ello. Así lo expresan los siguientes discursos:

“Lo que más más me marcó fue este fue el antes y después porque me tocó amortajarla [...] fue el impacto, dure como no sé cómo dos semanas soñando a la señora porque fue pues mi primer mi primer contacto" E6E

"Ver a una persona que tiene, a unos minutos acaba de estar bien, bueno viva y con signos vitales y de repente ver lo que ya no, como que de un segundo a otro te das cuenta que pues todo cambia en un segundo ¿no?, no nada uno lo tiene garantizado." E1L.

"Ese momento fue rápido, fue instantáneo y en ese momento yo no pude hacer nada después fue cuando intervine, eh, amortajando [...] esa fue mi intervención a lo mejor "E6E.

"De hecho estoy un poquito temblando, porque me hicieron recordar una experiencia muy fuerte [...] estoy hablando con, con sentimientos, con lo que yo en verdad sentí y pues ahorita me hicieron transportarme a ese día." E7A.

\section{DISCUSIÓN}

La experiencia vivida por los estudiantes frente a su primer contacto con la muerte de un paciente es dada en primer lugar por un impacto en la noticia del cese de la vida ya que este proceso causa a los profesionales y estudiantes de enfermería en concreto un impacto que influye en su actuar y su vida profesional ${ }^{(6)}$.

Esta primera impresión afecta a la forma de sentir preocupación sobre cómo se dará esta noticia y de su actuar ante esta situación, ya que no se sabe cómo reaccionar ante ella debido a que durante la transición de vida a muerte el personal de enfermería adopta diferentes mecanismos de defensa como el de justificar la muerte con la edad de la persona; cuando se trata del fallecimiento de un adulto mayor suele estar presente esta reacción ${ }^{(5)}$.

Independientemente de esto, cuando se es inexperto se reacciona de manera espontánea con diferentes emociones que se conjuntan en el impacto producido, llegando a colocarse como un espectador más del fenómeno al tratar de tener una explicación de lo que acontece. Esta primera reacción comienza configurando la experiencia y el contacto con la realidad vivida, generan en el estudiante una atención especial, pues es un fenómeno que impacta. 
Los estudiantes experimentan una serie de reacciones más específicas, como un bloqueo al no saber cómo intervenir, debido a que la muerte provoca un sin fin de emociones y sentimientos, algunos de ellos como miedo, angustia, incertidumbre y sufrimiento (21); los estudiantes expresan un encuentro emocional, una sensación de incertidumbre y con ello tratan de responder ante el miedo que le invade por las diversas reacciones, lo cual interfiere en el cuidado hacia el paciente y sus familiares, al no lograr expresar palabras y realizar acciones de apoyo. Algunas de estas reacciones son inesperadas al presenciar el morir, hay negación a la falta de respuesta del personal, ya que actúa de manera normalizada que a su vez fortalece la indiferencia, ensimismamiento y deshumanización ${ }^{(22) .}$

Los estudiantes difieren con la forma de actuar del profesional de enfermería, ya que algunos ejercen cuidados hacia el paciente fallecido marcados por una distancia, la literatura señala que esto puede ser una barrera emocional ante la situación (1), sin embargo, se necesitan estrategias para apoyar tanto al personal de enfermería a enfrentar estas situaciones con un sentido de humanismo, ya que los estudiantes aprenden de la dinámica cotidiana en la que se envuelven, por lo que las acciones de las enfermera son percibidas por ellos.

Todo lo anterior se conjunta en el estudiante, quien al ser su primer acercamiento busca cómo enfrentar el fenómeno de morir, pero resulta un reto más para este. En muchas ocasiones, los estudiantes no cuentan con las herramientas necesarias para enfrentar el proceso de muerte, afirmando que los planes de estudio están escasos en temas relacionados con muerte ${ }^{(6,23,24)}$, es por ello que surge inseguridad por creer que podrían ser juzgados por expresar sus emociones y por percibirse poco preparados para afrontar la muerte. Esta falta de preparación puede no ser generalizada en los planes de estudio, pero sí enfatiza en la necesidad de revisarlos e incluir herramientas y estrategias sobre la muerte, el cómo afrontarla y el cómo manejarla, no sólo centrándose en los cuidados post mortem.

Así mismo, lo estudiantes refirieron un tipo de "normalización" de la muerte y aunque es normal el proceso de morir, refirieron que los docentes les expresaban que era algo que se daría siempre, pero ellos lo percibían como un mensaje distante; no en tanto, con los conocimientos y recursos con los que disponen encontraron figuras de apoyo en el personal o profesores quienes sirven de sostén, brindando confianza y apoyo emocional, con base a su experiencia y conocimiento.

Así mismo, en ocasiones los docentes no brindan el apoyo necesario y se comportan de manera aprehensiva, a causa de que no tienen la formación necesaria para hablar sobre la muerte ${ }^{(1)}$, esto resulta relevante en el sentido de que el profesor es la figura formativa del estudiante y es quien da la base y soporte, no sólo académico, sino personal y humano, por lo que es imperante atender estas situaciones en los profesores y que estos transmitan al estudiante la confianza y una actitud de respeto y reflexión ante el proceso de morir. Algunos estudiantes refirieron por el contrario el apoyo del profesor que sumado al de algunas enfermeras sustenta la experiencia vivida en un entorno de confianza, seguridad, esperanza y consuelo.

Se requiere considerar la formación en la muerte que incluya a estudiantes y profesores en una dinámica relación de aprendizaje sobre el proceso de morir (25), aun así se referencia que el profesor sigue siendo una figura que puede apoyar en comprender el final de la vida del estudiante ${ }^{(2,24)}$, por esta razón es imperante que 
ambos aprendan y reflexionen en la práctica clínica sobre este fenómeno y que puedan generar significados que conlleven a intervenciones en favor de la persona fallecida, de la familia y de ellos mismos.

Aun y cuando los estudiantes se ven envueltos en diversas emociones intentan actuar con profesionalismo mediante lo aprendido durante su formación, buscan ofrecer un cuidado en la trascendencia entre la vida y la muerte, según Orozco-González, se actúa con humanismo, respeto y confidencialidad otorgando un cuidado digno (10); así mismo, con sus conocimientos figuran acciones que contribuyen en encontrar consuelo o satisfacción en lo realizado, de igual manera les dio esperanza de realizar un mejor cuidado en el futuro; debido a que los profesionales utilizan sus saberes desde sus vivencias sociales para encarar situaciones complicadas como lo es la muerte ${ }^{(26)}$.

Todo el fenómeno de la muerte, aunque es experimentado desde una falta de preparación, también es percibido como una experiencia de aprendizaje en el estudiante que le lleva a reflexionar sobre ello y lo que desencadena el compartirla, afirmando que no se sienten capaces de brindar apoyo a los familiares o al mismo paciente durante el proceso de morir, esto es ya un proceso reflexivo de las necesidades sentidas por el estudiante, por lo cual sería útil una mejor preparación para poseer conocimientos y actitudes basada en principios éticos ${ }^{(10)}$.

Por último, enmarcan estar envueltos en un tiempo que no conciben comprender ante la reacción del proceso de morir, por ello que enfermería necesita de mayor preparación para poder comprender lo inefable que es la trascendencia de vidamuerte y poder otorgar un cuidado espiritual, porque sin duda se tendrán dudas incomprensibles sobre lo que genera este proceso ${ }^{(22)}$, pero a pesar de esta percepción, sin duda, a los estudiantes les deja un gran aprendizaje basado en el humanismo y en el acercamiento hacia la persona fallecida, así como a la familia.

Los resultados se centran en la falta de formación por un lado que desencadena una serie de situaciones emocionales ${ }^{(25)}$, sin embargo, el estudiante es también un ser humano dotado de una capacidad reflexiva, lo que permite que enfrente el proceso desde una perspectiva humana, en donde el afecto, el humanismo y la dignidad humana son la fuente del aprendizaje del cuidado al final de la vida y de la muerte (2), por esta razón es necesario que como se evidencia en los discursos el estudiante tenga un espacio para reflexionar sobre la muerte y sobre lo que requiere en su formación para hacer frente a este proceso.

La fenomenología permite abordar esa esencia de la conciencia respecto a una experiencia vivida, la configura y dota al ser estudiante que la experimentó de una representación sobre lo vivido, el fenómeno de la muerte en la primera práctica sin duda configura la vida académica del estudiante ${ }^{(11)}$, pero también de sus profesores, del paciente fallecido en su dignidad como persona y de la familia que en ese momento atravesará por un momento complicado. El estudiante se configura además como ser humano con un sentido de humanismo que seguramente reflejará en sus próximas experiencias.

El fenómeno de la muerte en la primera experiencia vivida hace notar la falta de preparación y conocimiento sobre la muerte, pero también la oportunidad para crecer como personas, resignificando la muerte y el proceso de morir. 
Las limitaciones del presente estudio fueron que debido a la pandemia por COVID-19 no fue posible la realización de las entrevistas presenciales, sin embargo, es también una fortaleza, pues se evidencia el uso de medios digitales para su realización y la rica información obtenida. Otra limitación es que los resultados no pueden generalizarse, pero sí sientan un punto reflexivo para considerar que la enseñanza del morir y la muerte no se centre solamente en cuidados posteriores al suceso o en conceptos solamente teóricos, sino en espacios reflexivos durante toda la formación del estudiante.

\section{CONCLUSIÓN}

Las experiencias de los estudiantes de enfermería demuestran que estos tienen dificultades para afrontar la muerte y que a lo largo de su vivencia presentan una sensación de bloqueo, acompañada de emociones, destacando el miedo como el principal obstáculo en el cuidado para el paciente fallecido y los familiares; pero a pesar de esto, no es un impedimento para brindar cuidados de calidad necesarios, en razón de que enfermería les ha aportado empoderamiento para querer llevar a cabo el afrontamiento.

El razonamiento continuo sobre la experiencia ante la muerte los hizo pensar que esta va más allá de algo intangible; por ello se considera que una preparación más profunda sobre el qué hacer ante el proceso de morir, puede ser favorable para su actuar como profesional; además reconocer la importancia pedagógica de las experiencias de los estudiantes en el fenómeno, observando y comprendiendo las necesidades de los futuros profesionales de enfermería para dar iniciativa a mejorar la formación en tanatología y el cuidado en el proceso de morir del paciente. Además, se recomienda que las instituciones o programas educativos incluyan apoyo psicológico a los estudiantes, a la par de la formación en estos contenidos.

\section{Agradecimientos}

El presente trabajo les brinda un profundo agradecimiento a los estudiantes que aceptaron participar e hicieron posible la realización de esta investigación, así mismo a la estudiante de enfermería de la Universidad Autónoma de Aguascalientes Karen Vianney Hurtado Vargas por su gran colaboración durante todo el proceso y elaboración de esta investigación; de la misma manera al pasante de enfermería Erick Camacho Tobón de la Universidad de Orizaba Veracruz, que en algún momento participó en la elaboración de esta.

\section{REFERENCIAS}

1. Freitas TLL, Banazeski AC, Eisele A, Souza EN, Bitencourt JVOV, Souza SS. La visión de la Enfermería ante el Proceso de Muerte y Morir de pacientes críticos: una revisión integradora. Enferm. Glob. 2016; 15(41): 322-334. Murcia; 2016. Available from: https://scielo.isciii.es/pdf/eg/v15n41/revision2.pdf

2. Solano SAA, Bazán SACL. Experiencias de las estudiantes de enfermería en el cuidado a la persona al final de la vida - Chiclayo, Perú. 2016. Acc Cietna. 2018; 5(1):51-62. Available from: https://doi.org/10.35383/cietna.v5i1.10 
3. Ortúzar MG. La definición de muerte desde las perpectivas filosóficas de Bernard Gert y Daniel Wikler. Rev Filos y Teoría Política. 2018; (31-32):112-124. Available from:

http://www.memoria.fahce.unlp.edu.ar/art revistas/pr.2563/pr.2563.pdf\%0Ahttp:// www.fuentesmemoria.fahce.unlp.edu.ar/art revistas/pr.2563/

4. Flor L, Arellano H. El significado de la muerte. Revista Digital Universitaria UNAM. 2006;7(8): $\quad 2-7 \quad$ Available from: http://www.revista.unam.mx/vol.7/num8/art66/ago art66.pdf

5. Valle-Figueroa MC, García-Puga JA, Quintana-Zavala MO, García-Pérez Y. Experiencia del profesional de enfermería ante la muerte y el proceso de morir en unidades de cuidado intensivo. Sanus. 2019;(11):19-31. Available from: https://sanus.unison.mx/index.php/Sanus/article/view/148/126

6. Marrero GCM, García HAM. Vivencias de las enfermeras ante la muerte. Una revisión. Ene. 2019; 13(2): 1-13 p. Available from: http://www.eneenfermeria.org/ojs/index.php/ENE/article/view/844

7. B García-Solís, E. Araujo-Nava, V García-Ferrer, García-Hernández M. Sentimientos que manifiestan las enfermeras ante la muerte de su paciente 2014. 48-59

Available

from: http://web.uaemex.mx/revistahorizontes/docs/revistas/Vol5/5 SENTIMIENTOS.pdf

8. González-Martínez A, Campo-Martínez G, Ríos-Díaz R, Sánchez-Báez S, Vásquez-Lizalde E, Gómez-Cardona JP, Rosales-Hernández VS. Miedo y actitudes ante la muerte en estudiantes de enfermería de una universidad pública de Aguascalientes, Lux Médica. 2019; 14(4). 77-83 Available from: https://revistas.uaa.mx/index.php/luxmedica/article/view/2015/1858

9. Espinoza-Venegas M, Luengo-Machuca L, Sanhueza-Alvarado O. Atitudes em profissionais de enfermagem chilenos para o cuidado no final da vida. Análise multivariada. Aquichan. 2016;16(4):430-46. Available from: https://doi.org/10.5294/aqui.2016.16.4.3

10. Orozco-González MA, Tello-Sánchez GO, Sierra-Aguillón R, Gallegos-Torres RM, Xeque-Morales ÁS, Reyes-Rocha BL, Zamora-Mendoza. Experiencias y conocimientos de los estudiantes de enfermería, ante la muerte del paciente hospitalizado. Enferm. univ. 2013;10(1):8-13. Available from: http://www.scielo.org.mx/pdf/eu/v10n1/v10n1a3.pdf

11. Palomar GMC, Romero PM, Pérez GE, Romero PMI. Educar para la vida y la muerte a los estudiantes de Enfermería: "del miedo al amor." Enfermería (Montev.)2020;9(1):63-79.

Available

from: http://dx.doi.org/10.22235/ech.v9i1.2167

12. Castillo SN. Fenomenología como método de investigación cualitativa: preguntas desde la práctica investigativa. 2021; 10(20): 7-18. Available from: http://relmis.com.ar/ojs/index.php/relmis/article/view/fenomenologia como metodo 1167

13. Cara RR. Un estudio fenomenológico acerca de las percepciones de los profesionales de enfermería sobre la muerte. Ene. 2020; 14(1): 1-14. Available from: https://scielo.isciii.es/pdf/ene/v14n1/1988-348X-ene-14-1-e1417.pdf

14. Fuster Guillen DE. Investigación cualitativa: Método fenomenológico hermenéutico. Propós. represent. 2019;7(1):201-229. Available from: http://dx.doi.org/10.20511/pyr2019.v7n1.267

15. Van Manen M. Investigación educativa y experiencia vivida. Idea Books. Barcelona, España; 2003. 
16. Martínez-Salgado C. El muestreo en investigación cualitativa: principios básicos y algunas controversias. Cien Saude Colet. 2012;17(3):613-619. Available from: https://doi.org/10.1590/S1413-81232012000300006

17. Minayo MCS. Sampling and saturation in qualitative research: consensuses and controversies. Rev Pesqui Qual. 2017;5(7):1-12. Available from: https://editora.sepq.org.br/rpq/article/view/82/59

18. Guerrero-Castañeda RF, Menezes TMO, Ojeda-Vargas MG. Características de la entrevista fenomenológica en investigación en enfermería. Rev Gauch Enferm. 2017;38(2):e67458. Available from: https://doi.org/10.1590/19831447.2017.02.67458

19. Ayala Carabajo R. La metodología fenomenológico-hermeneútica de M. Van Manen en el campo de la investigación educativa. Posibilidades y primeras experiencias. Rev Investig Educ RIE. 2008;26(2):409-430. Available from; https://www.redalyc.org/pdf/2833/283321909008.pdf

20. Reglamento de la Ley General de Salud en Materia de Investigación para la Salud. México: Cámara de Diputados del H. Congreso de la Unión; 2014. Available http://www.diputados.gob.mx/LeyesBiblio/regley/Reg LGS MIS.pdf

21. Muñoz-Pino PI. Experiencia de estudiantes de enfermería ante su primer encuentro de cuidado con enfermos terminales. Invest. educ. enferm. 2014; 32(1): 87-94. Available from: http://www.scielo.org.co/scielo.php?pid=S012053072014000100010\&script=sci ar ttext\&tlng=es

22. García RM. Factores asociados a la actitud del profesional de enfermería ante la muerte del paciente terminal. Cuid salud. 2018;3(1):277-87. Available from: https://revistas.urp.edu.pe/index.php/Cuidado y salud/article/view/1424/1320

23. Santiago MEDCF, Carvalho EM, Pessoa RDL. O Entendimento dos Estudantes de Enfermagem Acerca do Processo de Morrer e Morte. Revista Ensaios e Ciencia. 2019;23(2):126. Available from: https://doi.org/10.17921/14156938.2019v23n2p126-131

24. Borges MC, Gomes BLBD. Percepção dos acadêmicos de enfermagem quanto ao processo terminal da vida. Rev Enferm Contemp. 2017;6(1):49-55. Available from: https://doi.org/10.17267/2317-3378rec.v6i1.1183

25. Praxedes AM, Araújo JL, Nascimento EGC. A morte e o morrer no processo de formação do enfermeiro. Psicol Saúde Doenças. 2018;19(2):369-376. Available from: http://dx.doi.org/10.15309/18psd190216

26. Perdigon AGC, Strasser G, Perdigon AGC, Strasser G. El proceso de muerte y la enfermería: un enfoque relacional. Reflexiones teóricas en torno a la atención frente a la muerte. Physis Rev Saúde Coletiva. 2015;25(2):485-500. Available from: https://doi.org/10.1590/S0103-73312015000200009 